\begin{tabular}{|c|l|}
\hline Title & ON LARGE TIME BEHA VIOR OF GROWTH BY BIRTH AND SPREA D \\
\hline Author(s) & Giga, Y oshikazu \\
\hline Citation & Hokkaido University Preprint Series in Mathematics, 1107, 1-20 \\
\hline Issue Date & 2018-02-01 \\
\hline DOI & 10.14943/81735 \\
\hline Doc URL & http://hdl.handle.net/2115/68261 \\
\hline Type & bulletin (article) \\
\hline File Information & Giga20171129.pdf \\
\hline
\end{tabular}

Instructions for use 


\title{
ON LARGE TIME BEHAVIOR OF GROWTH BY BIRTH AND SPREAD
}

\author{
YOSHIKAZU GIGA
}

\begin{abstract}
This is essentially a survey paper on a large time behavior of solutions of some simple birth and spread models to describe growth of crystal surfaces. The models discussed here include level-set flow equations of eikonal or eikonal-curvature flow equations with source terms. Large time asymptotic speed called growth rate is studied. As an application, a simple proof is given for asymptotic profile of crystal grown by anisotropic eikonal-curvature flow.
\end{abstract}

\section{INTRODUCTION}

Equations describing front propagation or surface evolution are very important in various fields of science and technology. Let $\Gamma_{t}$ be a hypersurface in $\mathbf{R}^{N}$ depending on time $t$, which describes, for example wave front or crystal surface. For simplicity, $\Gamma_{t}$ is assumed to be closed so that it is the boundary of some bounded open set $D_{t}$. Let $V$ be the normal velocity of $\Gamma_{t}$ in the direction of $\mathbf{n}$, a unit normal vector field of $\Gamma_{t}$ outward from $D_{t}$. The evolution given by a constant speed is often called Huygens' principle. Its explicit form is

$$
V=\sigma \quad \text { on } \Gamma_{t},
$$

where $\sigma$ is a constant. This is a famous eikonal equation. To describe evolution of crystal surface, one has to consider anisotropy called kinetic anisotropy. It can be written

$$
V=M(\mathbf{n}) \sigma \quad \text { on } \quad \Gamma_{t},
$$

where $M(\mathbf{n})$ is a given positive function defined on a unit sphere. The function $M$ is called a mobility. We refer this equation anisotropic eikonal equation. These equations are equations for one parameter family $\left\{\Gamma_{t}\right\}$.

In modern materials sciences, one also has to consider the curvature effect. The evolution is given by

$$
V=a H+\sigma \quad \text { on } \quad \Gamma_{t}
$$

with $a \geq 0$, where $H$ is the $(N-1)$ times mean curvature in the direction of $\mathbf{n}$, i.e., $H=-\operatorname{div}_{\Gamma} \mathbf{n}$, where $\operatorname{div}_{\Gamma}$ denotes the surface divergence. If $\sigma=0$ and $a=1$, this equation is known as the mean curvature flow equation, which stems from materials science, and has been widely studied in mathematical community. Thus the equation (1.3) is often called the eikonal-curvature flow equation if $a>0$ and $\sigma \neq 0$.

2010 Mathematics Subject Classification. Primary 35B40, Secondary 35F21, 35K61, 35K65, 49L25, 53C44. 
In materials science, one has to consider another anisotropy not only kinetic anisotropy. It is given as an anisotropic mean curvature or weighted mean curvature. Let $\gamma$ be a given nonnegative function in $\mathbf{R}^{N}$ which is positively homogeneous of degree one, i.e., $\gamma(\lambda p)=\lambda \gamma(p)$ for all $\lambda>0$, $p \in \mathbf{R}^{N}$. The anisotropic mean curvature $H_{\gamma}$ is defined at least formally by

$$
H_{\gamma}=-\operatorname{div}_{\Gamma} \xi(\mathbf{n}), \quad \xi(p)=\nabla_{p} \gamma=\left(\partial \gamma / \partial p_{1}, \ldots, \partial \gamma / \partial p_{N}\right) .
$$

It is known as the first variation of the interfacial energy $\int_{\Gamma} \gamma(\mathbf{n}) \mathrm{d} \mathcal{H}^{N-1}$ with respect to a variation of hypersurface $\Gamma$, where $\mathcal{H}^{N-1}$ denotes the $N-1$ dimensional Hausdorff measure. If $\gamma(p)=|p|$ so that $\xi(\mathbf{n})=\mathbf{n}, H_{\gamma}$ is nothing but standard $H$. A typical anisotropic version of (1.3) is

$$
V=M(\mathbf{n})\left(a H_{\gamma}+\sigma\right) \quad \text { on } \Gamma_{t} .
$$

It is very fundamental to ask whether or not the initial value problem for these equations is uniquely solvable. More precisely, the problem is that for a given initial data $\Gamma_{0}$ find $\left\{\Gamma_{t}\right\}_{t>0}$ solving (1.4). If one considers the problem globally-in-time, the singularity may develop for some smooth initial data even for (1.1). Thus one needs some weak notion of the solution. A level-set formulation is by now standard to solve such a problem globally-in-time. A level-set equation for (1.4) is the equation for $u$ in $\mathbf{R}^{N} \times(0, \infty)$ such that each level-set of $u$ moves by (1.4). To fix the orientation, we take $\mathbf{n}=-\nabla u /|\nabla u|$, where $\nabla$ denotes the spatial gradient, i.e., $\nabla=\left(\partial_{x_{1}}, \ldots, \partial_{x_{N}}\right), \partial_{x_{j}}=\partial / \partial x_{j}$. For example, the level-set equation for (1.1) and (1.3) are

$$
\begin{aligned}
& u_{t}-\sigma|\nabla u|=0, \\
& u_{t}-|\nabla u|\left(a \operatorname{div}\left(\frac{\nabla u}{|\nabla u|}\right)+\sigma\right)=0,
\end{aligned}
$$

respectively, where $u_{t}=\partial u / \partial t$. Unfortunately, level-set equations are highly degenerate in parabolic sense because there is no diffusion in the direction of $\nabla u$. Fortunately, there is notion of viscosity solutions [8], [18] based on order-preserving structure to handle continuous but non $C^{1}$ solutions. It turns out that such a notion is adjustable to this setting. Here are typical results. We consider the initial value problem of the level-set equation for (1.4), namely,

$$
\begin{gathered}
u_{t}-M(\nabla u /|\nabla u|)(a \operatorname{div}(-\xi(-\nabla u /|\nabla u|))+\sigma)|\nabla u|=0 \quad \text { in } \quad \mathbf{R}^{N} \times(0, \infty), \\
\left.u\right|_{t=0}=u_{0} .
\end{gathered}
$$

We shall use a short-hand notation $\{u>\ell\},\{u<\ell\},\{u=\ell\}$ to represent sets $\{(x, t) \mid u(x, t)>\ell\},\{(x, t) \mid u(x, t)<\ell\}$ and $\{(x, t) \mid u(x, t)=\ell\}$, respectively.

Theorem 1.1. Assume that $a \geq 0$ and $\sigma \in \mathbf{R}$ and that $M \geq 0$ is continuous. Assume that $\gamma$ is convex and $\gamma(p)>0$ for $p \neq 0$. Assume that $u_{0} \in C\left(\mathbf{R}^{N}\right)$ equals constant $\alpha$ outside a ball. For $\gamma$ assume either

(a) (smoothness) $\gamma \in C^{2}\left(\mathbf{R}^{N} \backslash\{0\}\right)$

or

(b) (crystalline) $\gamma$ is piecewise linear. 
Then the following statements hold.

(Global solvability) The initial value problem for (1.7) admits a unique continuous viscosity solution globally-in-time which equals $\alpha$ outside some ball in each finite time interval $(0, T)$.

(Uniqueness of level-sets) The set $\{u<\ell\}$ (resp. $\{u>\ell\}$ ) depends only on $\left\{u_{0}<\ell\right\}$ (resp. $\left\{u_{0}>\ell\right\}$ ) and independent of the choice of $u_{0}$. The set $\{u=\ell\}$ is called the level-set flow solution of (1.4) with initial data $\Gamma_{0}=\left\{u_{0}=\ell\right\}$.

The assumption at space infinity does not restrict application if one considers a closed hypersurface. This statement for (a) was first proved around thirty years ago by [7] and simultaneously for the level-set mean curvature flow equation by [11] (corresponding the case $a=1, \sigma=0$ in (1.3)); see e.g. [18] for details of the theory as well as related references. The case (b) of crystalline is not a simple generalization because the equation is nonlocal like total variation flow. For $N=2$, this statement was proved by [14] in 2001, where more general $\gamma$ is treated. For higher dimension $N \geq 3$, it is quite recent that this statement was proved by [24], [25] based on the work of [16], [17] in the sprit of [13]. The crucial steps are comparison principle and approximation arguments to construct a solution. Independently, A. Chambolle et al. [6], [5] proved such a result for "convex" mobility but general convex $\gamma$ including (b) based on distance functions.

If one looks the level-set equations, each level-set propagates by a given propagation law or surface evolution equations. This is also considered as spreading effects. For example, if one considers (1.5), each level-set spreads horizontally with velocity $\sigma$. Consider a crystal surface so that $u$ is now the height of crystal. Assume that initially it is flat so that $u_{0}=0$. Then it does not grow just by spreading effect. One needs birth of crystal so that crystal grows. There are two typical mechanism of growth of crystal surface [3]. One is the two-dimensional nucleation. The crystal surface grows by external supply of crystal molecules for a flat surface. It grows by catching such molecules. It is easy to catch molecules at the place where the crystal shape is not flat, i.e., $\nabla u \neq 0$ because of existence of microscopic steps. However, in the place where the surface is flat, there are no way to catch molecules unless there are step sources. At a very initial stage of the twodimensional nucleation the step source catches crystal molecules so that a small disk-like island is formed at the step source on a flat face. Then this island grows by spreading and there occurs another birth of small disk-like island. It results a "wedding cake" consisting of several disks. This is a way of birth of new crystal surface in the two-dimensional nucleation.

The other mechanism of crystal growth is the spiral growth which is more popular. As pointed out in [3], a pair of spirals opposite orientation whose centers are very close essentially forms a small island just like two-dimensional nucleation [33], [36], [34].

There are several models describing birth and spread macroscopically [32]. If one fixes location of step source, it is of the form

$$
u_{t}+F\left(\nabla u, \nabla^{2} u\right)=r(x),
$$


where $u_{t}+F$ is the left-hand side of the level-set equation (1.7) and $r(x) \geq 0$ is positive where step source exists. The simplest model is

$$
u_{t}-\sigma|\nabla u|=c I(x), \quad I(x)= \begin{cases}1 & x=0 \\ 0 & x \neq 0\end{cases}
$$

with $c>0$. This model is actually proposed [35] to describe some hightemperature superconductor by approximating spiral growth on a crystal surface.

Our goal in this paper is to study the large-time behavior of the solution. We are especially interested in proving the asymptotic speed or the growth rate

$$
\lim _{t \rightarrow \infty} \frac{u(x, t)}{t}
$$

and its property. This is a very general question for partial differential equations of evolution type. There are by now several general theory for first order problems and some for second order problems (see review article [30]) but our problem is not covered by known theories like the weak KAM theory so far; see Subsection 2.3 and Subsection 5.1. The next result is a straightforward generalization of the result of [23].

Theorem 1.2 (Existence of asymptotic speed). Assume that $r \geq 0$ is Lipschitz (continuous) and compactly supported. Assume the same assumption in Theorem 1.1 on (1.7) with $a \geq 0, \sigma>0$ and $M>0$. Assume further that $\gamma \in C^{2}\left(\mathbf{R}^{N} \backslash\{0\}\right)$. Let $u$ be the viscosity solution of (1.8) having the same left-hand side as (1.7) with $u_{0}=0$. Then the asymptotic speed $R=\lim _{t \rightarrow \infty} u(x, t) / t$, which is nonnegative, exists and the convergence is locally uniform.

Let $1_{E}$ denote the characteristic function of $E$, i.e.,

$$
1_{E}(x)= \begin{cases}1, & x \in E \\ 0, & \text { otherwise. }\end{cases}
$$

If $r_{\varepsilon}(x)$ is close to $c 1_{E}$ in the sense $r_{\varepsilon}=c \eta_{\varepsilon} * 1_{E}$, where $\eta_{\varepsilon}$ is the Friedrichs mollifier i.e., $\eta \in C_{c}^{\infty}\left(\mathbf{R}^{N}\right), 0 \leq \eta \leq 1, \int \eta \mathrm{d} x=1 \eta(x) \equiv 0$ for $|x| \geq$ $3 / 4$ and $\eta_{\varepsilon}(x)=\varepsilon^{-N} \eta(x / \varepsilon)$, one might expect the asymptotic speed $R_{\varepsilon}$ for $r_{\varepsilon}$ converges to $c$ as $\varepsilon \downarrow 0$. This is true for the first order model like $u_{t}-\sigma|\nabla u|=r(x)$. Unfortunately, this is not true in general for the secondorder models. The next result easily follows by the comparison principle from similar results in [22], where the case $r=c 1_{E}$ is considered. For this non-Lipschitz $r$, we do not know even the existence of asymptotic speed.

Theorem 1.3. Consider (1.8) with $r=r_{\varepsilon}$ in the plane. Assume the lefthand side is the same as (1.6) with $a=\sigma=1$ and that $\gamma(p)=|p|, M(p)=$ $|p|$. Assume that $E$ is a closed square whose edge length is $2 d$ with $d \in$ $(1 / \sqrt{2}, 1)$ so that $E$ is not contained in nor not contains a unit disk. Then $0<\liminf _{\varepsilon \rightarrow 0} R_{\varepsilon} \leq \limsup _{\varepsilon \rightarrow 0} R_{\varepsilon}<c$.

This is because of curvature effect of spreading. There are few literature on asymptotic speed of second-order problems, for example, the work [38], [39] studied the turbulent flow speed for what is called G-equations. 
Our next concern is the asymptotic shape. For this purpose, we introduce a notion of the support function $W_{M}$ of the polar of $1 / M$, i.e.,

$$
W_{M}(x)=\sup \{x \cdot p|| p \mid \leq 1 /(M(p /|p|))\}, x \in \mathbf{R}^{N} .
$$

Its one sub level-set is often called the Wulff shape

$$
\begin{aligned}
\mathcal{W}_{M} & =\left\{x \in \mathbf{R}^{N} \mid W_{M}(x) \leq 1\right\} \\
& =\bigcap_{|m|=1}\left\{x \in \mathbf{R}^{N} \mid x \cdot m \leq M(m)\right\} .
\end{aligned}
$$

Theorem 1.4 (Asymptotic profile). Let $u$ be as in Theorem 1.2. Then

$$
\lim _{\lambda \rightarrow \infty} \frac{u(\lambda x, \lambda t)}{\lambda}=R\left(t-W_{M}(x) / \sigma\right)_{+}
$$

locally uniformly for $(x, t) \in \mathbf{R}^{N} \times(0, \infty)$, where $b_{+}=\max (b, 0)$.

Remark 1.5. The results in Theorem 1.2 and Theorem 1.4 can be easily extended for general bounded uniformly continuous initial data by simple comparison with constant initial data. Thus $R$ is independent of $u_{0}$.

As a byproduct of our analysis, we give a simple new proof of anisotropic profile of level-set flow of (1.4) when the shape is growing. Such a result is originally proved by H. Ishii, G. E. Pires and P. E. Souganidis [29] a long time ago. From the point of (1.8), it is asymptotic profile of the horizontal growth.

Theorem 1.6. Let $\Gamma_{t}$ be the level-set flow solution in Theorem 1.1. Assume that $\gamma \in C^{2}\left(\mathbf{R}^{N} \backslash\{0\}\right)$. Assume that $\Gamma_{0}$ strictly encloses $(a(N-1) / \sigma) \mathcal{W}_{\gamma}$ up to translation. Then $\Gamma_{t} / t \rightarrow \partial \mathcal{W}_{M}$ as $t \rightarrow \infty$ in the sense of the Hausdorff distance.

Note that our assumption for $\Gamma_{0}$ is weaker than [29, Theorem 6.1] where they assured $\Gamma_{0}$ encloses a sufficiently large ball. Note that their proof based on characterization of $\lim _{t \rightarrow \infty} u(t x, t)$ works even when $\gamma$ is crystalline, where $u$ is in Theorem 1.1. For crystalline case evolution of a convex shape by (1.4) is analyzed in [15], where the role of anisotropy in $M$ and $\gamma$ is clarified. We expect that all results in Theorem 1.2 - Theorem 1.6 can be extended to crystalline $\gamma$ if appropriate stability holds (See Subsection 5.2).

This paper is organized as follows. In Section 2 we discuss the first-order model while in Section 3 we discuss the second-order model and give a sketch of the proof of Theorem 1.2. In Section 4 we prove Theorem 1.3, Theorem 1.4 and Theorem 1.6. In Section 5 we discuss unscaled asymptotic profiles and open problems.

\section{First ORDER MODELS}

We consider

$$
u_{t}-\sigma|\nabla u|=r(x)
$$

or its anisotropic version

$$
u_{t}-\sigma M(-\nabla u)=r(x), \quad\left(M(p):=M(p /|p|)|p| \text { for } p \in \mathbf{R}^{N}\right)
$$


for a bounded upper semicontinuous function $r$. Here $M$ is assumed to be continuous and nonnegative and $\sigma>0$. If $r$ is continuous, the standard theory of viscosity solutions yields a unique global-in-time solution for any bounded uniformly continuous initial data. However, if $r$ is not continuous, typically $r(x)=c I(x)$, the solution may not be unique. We need to consider a kind of maximal solution which is formulated as an envelope solution in [19]. If one applies this equation to describe height of crystal surface by $u$, then it seems to be natural to consider a maximal solution [35]. In fact, there exists a unique global-in-time envelope solution for any such $r$ when initial data $u_{0}$ is bounded uniformly continuous [19, Theorem 3.20]. Note that $M$ doesn't need to be convex. We shall discuss several explicit solutions.

2.1. Explicit solutions. We consider (2.1) with $r(x)=c I(x), \sigma>0$ with initial data $u_{0}=0$.

Proposition 2.1. Assume that $\sigma>0$.

(i) If $c>0$, then $u_{R}(x, t)=R(t-|x| / \sigma)_{+}$for $0 \leq R \leq c$ is a viscosity solution of (1.9) with $\left.u_{R}\right|_{t=0}=0$. The solution $u_{c}$ is the unique envelope solution with zero initial data.

(ii) If $c \leq 0$, then $u \equiv 0$ is a viscosity solution of (1.9) with initial data $\left.u\right|_{t=0}=0$ (It is actually the unique viscosity solution.)

Proof. (i) It is rather trivial to see that $u_{R}$ solves (1.9) except $x=0$. At the origin assume that $u_{R}-\varphi$ takes its maximum at $(0, \hat{t}), \hat{t}>0$ for some $C^{1}$ function $\varphi$. Then $\varphi_{t}(0, \hat{t}) \leq R$ so that

$$
\varphi_{t}(0, \hat{t})-\sigma|\nabla \varphi(0, \hat{t})|-c I(0) \leq R-c \leq 0 .
$$

Thus, $u_{R}$ is a subsolution. Note that at $t=|x| / \sigma$, there is no way to touch from above. The test from below at $t=|x| / \sigma$ yields that $u_{R}$ is a supersolution. We thus conclude that $u_{R}$ is a solution.

(ii) This is very easy to check, so the proof is safely left to the reader.

Its anisotropic version is as follows. We consider (2.2) with $r(x)=c I(x)$.

Proposition 2.2. Assume that $\sigma>0$.

(i) If $c>0$, then $u_{R}(x, t)=R\left(t-W_{M}(x) / \sigma\right)_{+}$for $0 \leq R \leq c$ is a viscosity solution of

$$
u_{t}-\sigma M(-\nabla u)=c I(x)
$$

with $\left.u_{R}\right|_{t=0}=0$. The solution $u_{c}$ is the unique envelope solution starting from zero.

(ii) If $c \leq 0$, then $u \equiv 0$ is a viscosity solution of (2.3) with $\left.u\right|_{t=0}=0$.

The proof of this Proposition 2.2 is of course more involved. However, if one notices that $M\left(\nabla W_{M}\right)=1$, it is rather easy.

2.2. Asymptotic speed and profile. The next result is a special case of [27, Theorem 2.3]. For $u: \mathbf{R}^{N} \times(0, \infty) \rightarrow \mathbf{R}$, let $u^{\lambda}(x, t)$ be a rescaled function defined by

$$
u^{\lambda}(x, t)=u(\lambda x, \lambda t) / \lambda \text { for } \quad \lambda>0 .
$$


Theorem 2.3. Assume that $r \geq 0$ is continuous and compactly supported. Let $u$ be the viscosity solution of (2.2) with initial data $\left.u\right|_{t=0}=0$. Let $c=\max r(x)$. Then $u^{\lambda} \rightarrow c\left(t-W_{M}(x) / \sigma\right)_{+}$locally uniform as $\lambda \rightarrow \infty$.

Proof. The proof given in [27] is studying relaxed upper and lower limit. We here give a simple proof. We may assume that zero is a maximum point of $r$ by translation. We know the solution is Lipschitz independent of regularity of $r$ if initial data is Lipschitz [19] since the Hamiltonian is coercive in the sense that $M(p) \rightarrow \infty$ as $|p| \rightarrow \infty$. Thus $\left\{u^{\lambda}(x, t)\right\}$ is equi-Lipschitz in $\mathbf{R}^{N} \times(0, T)$. By the Ascoli-Arzelà theorem for each sequence, there is a convergent subsequence $u^{\lambda_{i}}$ and limit $v$ such that $v^{\lambda_{i}} \rightarrow v$ locally uniformly (by diagonal argument) as $\lambda \rightarrow \infty$. By the stability of viscosity solutions [18], $v$ satisfies (2.3) with $c=\max r(x)$.

Fortunately, $r_{\lambda}(x) \geq c I(x)$ for $r_{\lambda}(x)=r(\lambda x)$ so $v$ must be the envelope solution of (2.3) and it must be unique. Thus, the convergence $u^{\lambda} \rightarrow v$ becomes full convergence and $v(x)=c\left(t-W_{M}(x) / \sigma\right)_{+}$.

This statement is not exactly contained in Theorem 1.4 where $r$ is assumed to be Lipschitz. This asymptotic results yield asymptotic speed as a Corollary.

Corollary 2.4. Under the same assumption of Theorem 2.3, the asymptotic speed

$$
\lim _{t \rightarrow 0} \frac{u(x, t)}{t}=c
$$

exists and it is equals to $\max r$.

This is easy to prove by taking $t=1, \lambda=t, x=x / t$. Note that the asymptotic speed is nothing but the maximum of $r$. For the first-order problem, the situation like Theorem 1.3 does not occur.

2.3. Non-coercive case. For a coercive case, large-time behavior is well studied. It goes back to the work of G. Namah and J.-M. Roquejoffre [31] and A. Fathi [12]. It gives even asymptotic expansion $u(x, t) \sim c t+w(x)$ in the sense for a given ball $B$

$$
\sup _{x \in B}|u(x, t)-c t-w(x)| \rightarrow 0 \quad \text { as } \quad t \rightarrow \infty .
$$

Here $w$ is a viscosity solution of a cell problem.

$$
c-M(-\nabla w)=r(x) .
$$

Solutions may not be unique because the set $\{\hat{x} \mid \max r=r(\hat{x})\}$ plays a role of Aubry set. See Section 5. We do not touch this problem. We say the equation (1.8) of the form

$$
u_{t}+F(\nabla u)=r(x)
$$

is coercive if

$$
\lim _{|p| \rightarrow \infty} F(p)=-\infty .
$$

We notice that if the problem is non-coercive, the large-time behavior is not well studied although there are several works by [40], [20], [21] related to 
crystal growth. For example, if

$$
u_{t}-\frac{|\nabla u|}{|\nabla u|+1}=r(x),
$$

it is not yet clear what the asymptotic speed is. Moreover, for a constant $c>0$, the uniqueness of a solution with $r=c I(x)$ is not guaranteed. In fact, according to [19, Example 5.15]

$$
U_{c}(x, t)= \begin{cases}c t-\frac{c}{1-c}|x| & \left(|x| \leq(1-c)^{2} t\right) \\ \left((\sqrt{t}-\sqrt{|x|})_{+}\right)^{2} & \left(|x| \geq(1-c)^{2} t\right)\end{cases}
$$

is a unique envelope solution of (2.4) with $r=c I(x)$ when $c \leq 1$. However, if $c>1$ even an envelope solution may not be unique.

\section{SECOND ORDER MODELS}

3.1. Models. We now consider the equation (1.8) for (1.7), namely

$$
u_{t}-M(-\nabla u)(a \operatorname{div}(-\xi(-\nabla u))+\sigma)=r(x) \text {. }
$$

The major difference from (2.2) is that the curvature effect is included in spreading process; see Figure 1 for the graph of $u$ governed by (3.1). In particular, if the radius of island is too small, it does not spread. In the two-dimensional nucleation, it is more realistic to consider the case that the place of birth may depend on time, i.e., $r$ may depend on $t$. However, in this note we only consider the case when $r$ is independent of time because it is already complicated than what we expect. Moreover, if one uses this model to describe the spiral growth, $r$ must be independent of time and this is better approximation than (2.2).

We first recall the well-posedness of the initial value problem for (3.1).

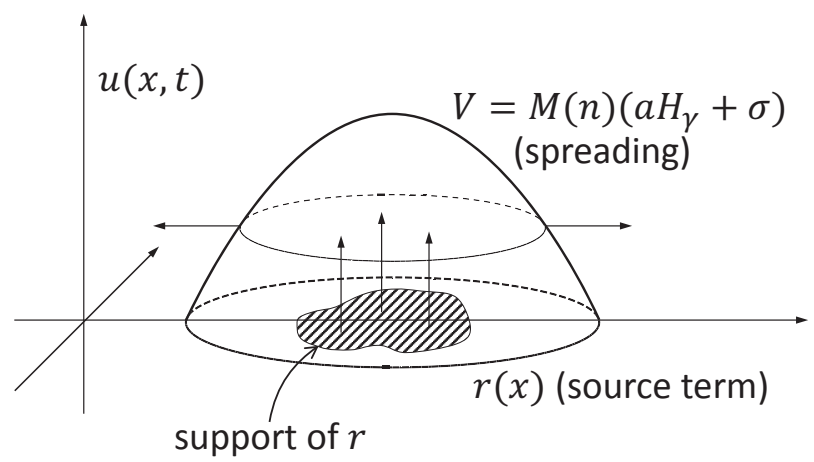

Figure 1. The graph of $u$ at time $t$ solving (3.1)

Theorem 3.1 (Solvability). Assume the same hypotheses of Theorem 1.1 concerning $a, \sigma, M, \gamma$ and $u_{0}$. Assume that $r$ is continuous and has compact support. Then the initial value problem for (3.1) with $\left.u\right|_{t=0}=u_{0}$ admits a unique continuous viscosity solution u globally-in-time which equals $\alpha$ outside some ball in each finite time interval. 
Proof. The proof for the case when $\gamma$ is $C^{2}$ outside the origin is by now standard and well known as in [18]. However, the case when $\gamma$ is crystalline is quite new even if $r=0$ [24], [25]. We first prove the comparison principle. Suppose that $u$ is viscosity subsolution and $v$ is a viscosity supersolution as in [24], [25]. Then we have to conclude that $u \leq v$ if initially $u \leq v$. The definition for viscosity sub and supersolution with $r \not \equiv 0$ is not given there but it is obtained as a trivial modification. This comparison principle can be proved along the line of [24], [25] if one replaces doubling variable procedure with shift parameter $\zeta$ by

$$
\Phi_{\zeta}=u(x, t)-v(y, s)-\frac{|x-y-\zeta|^{2}}{2 \varepsilon}-S_{\varepsilon, \delta}(t, s),
$$

where $S_{\varepsilon, \delta}(t, s)$ should be

$$
S_{\varepsilon, \delta}(t, s)=\frac{|t-s|^{2}}{2 \varepsilon}+\frac{\delta}{T-t}+\frac{\delta}{T-s} ;
$$

in [24], [25] $\delta$ is taken to be equal to $\varepsilon$. We fix $\delta>0$ small enough as in [13] unrelated to $\varepsilon$. We argue by contradiction as in [24], [25] and end up with

$$
\frac{2 \delta}{T^{2}} \leq \frac{\delta}{(T-\hat{t})^{2}}+\frac{\delta}{(T-\hat{s})^{2}} \leq r(\hat{x})-r(\hat{y}),
$$

where $(\hat{x}, \hat{t}, \hat{y}, \hat{s})$ is a maximum point of $\Phi_{\zeta}$ for small $\zeta$ depending on $\varepsilon$. If $\varepsilon \rightarrow 0$ with $\zeta \rightarrow 0$, we observe $\hat{x}-\hat{y} \rightarrow 0$ so we get a contradiction. Existence can be proved by approximation as in [24], [25].

3.2. Radial case and its generalization. We consider the special case when $M=\gamma$. In other words, kinetic anisotropy agrees with interfacial anisotropy. Moreover, assume that $r$ depends only on $W_{\gamma}(x)$, i.e., $r(x)=$ $h\left(W_{\gamma}(x)\right)$, We postulate that the solution $u(x, t)$ only depends on $W_{\gamma}(x)$, i.e., $u$ has the form

$$
u(x, t)=U\left(W_{\gamma}(x), t\right) .
$$

Since we know that $\xi\left(\nabla W_{\gamma}(x)\right)=x /|x|$ so that $\operatorname{div} \xi\left(W_{\gamma}(x)\right)=N-1$ and $\gamma\left(\nabla W_{\gamma}\right)=1$,

$$
u_{t}-\gamma(-\nabla u)(a \operatorname{div}(-\xi(-\nabla u))+\sigma)=h\left(W_{\gamma}(x)\right)
$$

is reduced to

$$
U_{t}-\frac{a(N-1)}{\rho} U_{\rho}+\sigma U_{\rho}=h(\rho)
$$

if $U=U(\rho, t)$ is a nonincreasing function with respect to $\rho$, i.e., $U_{\rho} \leq 0$. The equation for $U$ is the same as in radial solution for isotropic case. The equation (3.3) is now linear first order but non-coercive Hamilton-Jacobi equation with singularity.

To see that the asymptotic speed may not be $\max h$, we give a few examples. We rather consider discontinuous $h$ of the form

$$
h=c 1_{\left[0, \rho_{0}\right]} .
$$

Since the right-hand side is not continuous, we do not expect uniqueness of viscosity solutions. We rather consider the maximal solution. We set a critical number

$$
\rho_{*}=a(N-1) / \sigma
$$


and define

$$
\psi_{0}(\rho)=c\left\{\rho+\rho_{*} \log |\sigma \rho-a(N-1)|\right\} / \sigma=c\left\{\rho+\rho_{*} \log \left|\sigma\left(\rho-\rho_{*}\right)\right|\right\} / \sigma,
$$

which solves

$$
\left(-\frac{a(N-1)}{\rho}+\sigma\right) \partial_{\rho} \psi=c \quad \text { for } \quad \rho \neq \rho_{*} .
$$

Moreover, $\partial_{\rho} \psi_{0}(0)=0$ as we expected.

Theorem 3.2. Consider (3.2) with (3.4) and $\left.u\right|_{t=0}=0$. Assume that $\gamma$ is $C^{2}$ outside the origin or that $\gamma$ is crystalline with $N=2$. Assume that $\sigma>0, a>0$.

(i) If $\rho_{0}<\rho_{*}$, then

$$
u(x, t)= \begin{cases}\min \left\{\psi\left(W_{\gamma}(x)\right)_{+}, c t\right\} & \text { for } x \text { with } W_{\gamma}(x)<\rho_{*} \\ 0 & \text { for } x \text { with } W_{\gamma}(x) \geq \rho_{*}\end{cases}
$$

with $\psi(\rho)=\psi_{0}(\rho)-\psi_{0}\left(\rho_{0}\right)$ is the maximal viscosity solution.

(ii) If $\rho_{0}>\rho_{*}$, then

$$
u(x, t)= \begin{cases}\min \left\{c t,\left(c t-\psi\left(W_{\gamma}(x)\right)\right)_{+}\right\} & \text {for } x \text { with } W_{\gamma}(x) \geq \rho_{*} \\ c t & \text { for } x \text { with } W_{\gamma}(x)<\rho_{*}\end{cases}
$$

is the maximal viscosity solution.

(iii) If $\rho_{0}=\rho_{*}$, then

$$
u(x, t)=c t 1_{W_{\gamma}}
$$

is the maximal viscosity solution.

From this we see the growth speed depends on geometry where $u$ takes maximum. This is quite different from the first-order model. In fact in the case (i) $u(x, t)$ is bounded as $t \rightarrow \infty$ and $\lim _{t \rightarrow \infty} u(x, t) / t=0$. In the case (ii) $c=\lim _{t \rightarrow \infty} u(x, t) / t$ for all $x \in \mathbf{R}^{N}$ while in the case (iii) $c=\lim _{t \rightarrow \infty} u(x, t) / t$ for $x \in \rho_{*} \mathcal{W}_{\gamma}$ while outside $\rho_{*} \mathcal{W}_{\gamma}$ we observe that $u(x, t) \equiv 0$. See Figure 2 for profiles of the graph of $u$ at time $t$.
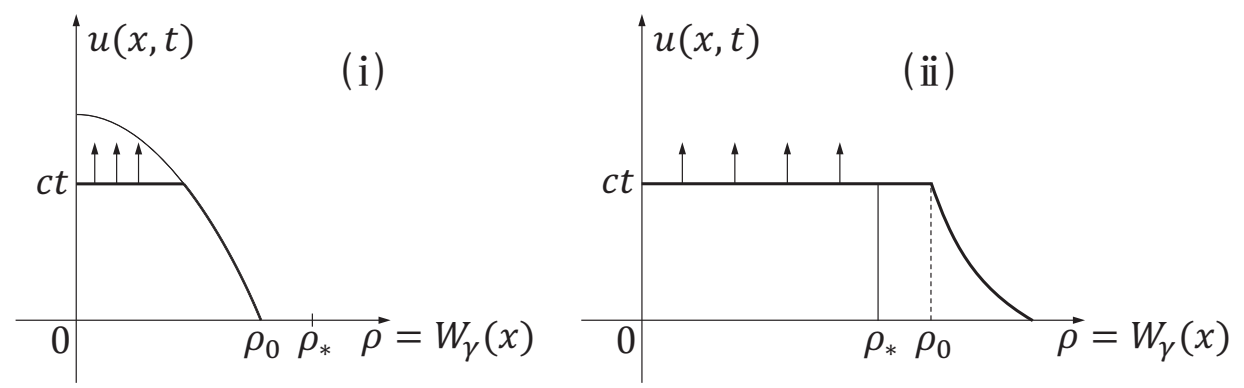

Figure 2. The graph of $u$ at time $t$

By the way, the function $U(\rho, t)=\min \{\psi(\rho)$, ct $\}$ solves (3.3) with (3.4) for $\rho \in\left(0, \rho_{*}\right), t \in \mathbf{R}$ if $\rho_{0}<\rho_{*}$ while $U(\rho, t)=\min \{c t, c t-\psi(\rho)\}$ solves (3.3) with (3.4) for $\rho>\rho_{*}, t \in \mathbf{R}$ if $\rho_{0}>\rho_{*}$ in viscosity sense. If one omits the plus part symbol in (i), (ii), then $u$ is an entire viscosity solution, i.e., it solves (3.1) for all $t \in \mathbf{R}, x \in \mathbf{R}^{N}$; in the case of (i) one has to exclude the place where $\rho=\rho_{*}$. 
The results in Theorem 3.2 is essentially proved in [22], where the isotropic case, i.e., $\gamma(p)=|p|$ or $W_{\gamma}(x)=|x|$ is discussed. Theorem 3.2 is a trivial extension of results in [22, Sect. 4] to anisotropic case. For crystalline case, this result should be true. Although it is easy to see that the proposed solution is a viscosity solution, to show the maximality we need some stability of crystalline level-set equation which is so far not available for $N \geq 3$; see Section 5.2. The case $N=2$ is proved in [14].

3.3. Lipschitz bounds. We shall derive Lipschitz bounds in time and space for (3.1) when the initial data $u_{0}=0$ and $r \geq 0$ is Lipschitz. These are straightforward extension of those in [23], where the isotropic case is discussed.

Lemma 3.3 (Bound for time derivative). Assume the same hypotheses of Theorem 3.1 concerning $a, \sigma, M, r$. Assume that $u_{0}=0$ and $r \geq 0$. Let $u$ be the viscosity solution of (3.1) in Theorem 3.1. Then $u$ is Lipschitz in $t$ and

$$
0 \leq u_{t}(x, t) \leq c:=\max _{\mathbf{R}^{N}} r(x)
$$

for all $x \in \mathbf{R}^{N}$ and almost all $t \geq 0$.

Proof. Since $v(x, t)=c t$ is a viscosity supersolution (see e.g. [18]) and $w \equiv 0$ is a viscosity supersolution of (3.1), by the comparison principle we easily see that

$$
0 \leq u(x, t) \leq c t \quad \text { in } \quad \mathbf{R}^{N} \times[0, \infty)
$$

For any given $s>0$, both

$$
u^{s}(x, t):=u(x, t+s) \text { and } u(x, t)
$$

are viscosity solutions of $(3.1)$. Since $u^{s}(x, 0) \geq u(x, 0)=u_{0}=0$, by the comparison principle we obtain

$$
0 \leq\left(u^{s}-u\right)(x, t) \leq\left.\sup _{\mathbf{R}^{N}}\left(u^{s}-u_{0}\right)\right|_{t=0} \leq c s
$$

which yields the desired estimate.

Lemma 3.4 (Bound for spatial derivative). Assume the same hypotheses of Lemma 3.3. Assume furthermore that $\gamma \in C^{2}\left(\mathbf{R}^{N} \backslash\{0\}\right)$ and that $r$ is Lipschitz. Then u is spatially Lipschitz and it gradient is essentially bounded. More precisely, its $L^{\infty}$-norm has a bound

$$
\|\nabla u\|_{L^{\infty}\left(\mathbf{R}^{N}\right)}(t) \leq K
$$

with $K$ independent of $t \in(0, \infty)$.

This can be proved by what is called Bernstein's method. We first recall a simple matrix inequality.

Lemma 3.5. Let $A$ and $B$ be real symmetric matrices. Assume that $A$ is nonnegative definite, i.e., $A \geq 0$. Then

$$
(\operatorname{tr} A B)^{2} \leq \operatorname{tr}(A B B) \operatorname{tr} A .
$$


This follows from the Schwarz inequality

$$
\left(\operatorname{tr}\left({ }^{t} a b\right)\right)^{2} \leq \operatorname{tr}^{t} a a \operatorname{tr}^{t} b b
$$

for general real square matrices $a, b$ by setting $a=A^{1 / 2}, b=A^{1 / 2} B$, where ${ }^{t} a$ denotes the transpose of $a$.

Formal proof of Lemma 3.4. We write (3.1) in the form of (1.8) with $F=$ $F(p, X)$. Pretending that everything is smooth, we differentiate (1.8) in $x_{k}$ to get

$$
u_{k t}+\sum_{\ell=1}^{N} \frac{\partial F}{\partial p_{\ell}} u_{k l}+\sum_{i, j=1}^{N} \frac{\partial F}{\partial X_{i j}} u_{k i j}=r_{k},
$$

where we use a shorthand notation $u_{k}=\partial_{x_{k}} u, u_{k \ell}=\partial_{x_{k}} \partial_{x_{\ell}} u$ and so on. We multiply $u_{k}$ and add from 1 to $N$ to get differential inequality for $w=$ $\sum_{k=1}^{N} u_{k}^{2} / 2$ of the form

$$
w_{t}+\sum_{\ell=1}^{N} \frac{\partial F}{\partial p_{\ell}} w_{\ell}+\sum_{\ell, i, j=1}^{N} \frac{\partial F}{\partial X_{i j}}\left(w_{i j}-u_{j \ell} u_{i \ell}\right)=\nabla r \cdot \nabla u .
$$

We set

$$
a_{i j}=-\frac{\partial F}{\partial X_{i j}}=a M(-p) \frac{\partial^{2} \gamma}{\partial p_{i} \partial p_{j}}(-p) \quad \text { with } \quad p=\nabla u .
$$

By Lemma 3.5 we observe that

$$
\sum_{i, j, \ell}^{N} a_{i j} u_{j \ell} u_{i \ell} \geq\left(\sum_{i, j} a_{i j} u_{i j}\right)^{2} / \sum_{i} a_{i i} \geq\left(\sum_{i, j} a_{i j} u_{i j}\right)^{2} / A
$$

with some constant $A>0$ independent of $p$ since $\sum_{i} a_{i i} \leq A$. We now obtain

$$
w_{t}+\sum_{\ell} \frac{\partial F}{\partial p_{\ell}} w_{\ell}-\sum_{i, j} a_{i j} w_{i j}+\left(\sum_{i, j} a_{i j} u_{i j}\right)^{2} / A \leq \nabla r \cdot \nabla u .
$$

Since the equation (1.8) is quasilinear, we observe that

$$
\sum_{i, j} a_{i j} u_{i j}=u_{t}-\sigma M(-\nabla u)-r .
$$

Since $\left|u_{t}\right| \leq c$ and $\sigma M(p) \geq m_{0}|p|$ with some constant $m_{0}>0$, we see that

$$
\left(\sum_{i, j} a_{i j} u_{i j}\right)^{2} \geq\left(m_{0}|\nabla u|-c\right)^{2} \geq \frac{m_{0}}{2}|\nabla u|^{2}-c^{2} \quad \text { if } \quad m_{0}|\nabla u|>c .
$$

Let $\beta_{0}$ be the largest zero of

$$
\left(\frac{m_{0}}{2} \beta^{2}-c^{2}\right) / A-\|\nabla r\|_{L^{\infty}\left(\mathbf{R}^{N}\right)} \beta=0 .
$$

We thus conclude that

$$
w_{t}+\sum_{\ell} \frac{\partial F}{\partial p_{\ell}} w_{\ell}-\sum_{i, j} a_{i j} w_{i j} \leq 0 \quad \text { if } \quad|\nabla u| \geq \max \left(\beta_{0}, c / m_{0}\right)=: \beta_{1} .
$$


By the comparison principle (assuming that the space infinity is well controlled), we observe that $w \leq \beta_{1}^{2} / 2$. We now obtain the desired bound $K=\beta_{1}$ at least formally.

To realize the idea, we fix time and approximate the equation so that the singularity near $\nabla u=0$ zero is removed and that the problem is uniformly elliptic to get a smooth solution. We have skipped all this procedure and have left the details to [23].

3.4. Existence of asymptotic speed. We are in position to prove the existence of asymptotic speed (Theorem 1.2). For this purpose, we check the motion of the top. We set

$$
m(t)=\sup _{x \in \mathbf{R}^{N}} u(x, t) .
$$

Lemma 3.6. Assume the same hypothesis of Theorem 1.2. Then $m(t)$ is subadditive and $R:=\lim _{r \rightarrow \infty} m(t) / t$ exists and equals $\inf _{t>0} m(t) / t$ with $R \in[0, \infty)$.

Proof. Since $v(x, t)=u^{s}(x, t)-m(s)$ is a subsolution of (3.1) with $u^{s}(x, t)=$ $u(x, t+s)$ for $s>0$ and since $v(x, 0) \leq u(x, s)-m(t) \leq 0=u(x, 0)$, by the comparison principle we see that $v(x, t) \leq u(x, t)$ in $\mathbf{R}^{\bar{N}} \times(0, \infty)$. Take sup in both sides in $x$ to get

$$
m(t+s)-m(s) \leq m(t)
$$

which implies the subadditivity. The other assertion follows by Fekete's lemma (see e.g. [1, p. 95] for the proof) for a subadditive function.

In this argument, we do not use Lipschitz bound so Lemma 3.6 is still valid for continuous $r$. Also it applies to the case of crystalline.

Proof of Theorem 1.2. Since $u \geq 0$, if $R=0$ in Lemma 3.6, the convergence

$$
R=\lim _{t \rightarrow \infty} u(x, t) / t
$$

immediately follows with $R=0$. We may assume that $R>0$.

It suffices to prove that for a given ball $B$ and $\varepsilon>0$ there exists $T$ such that

$$
u(x, t) / t \geq R-\varepsilon \text { for } t>T, x \in B .
$$

We may assume that $B$ includes $\operatorname{supp} r$, the support of $r$. Assume that $x_{t}$ is the maximizer of $u(x, t)$, i.e., $u\left(x_{t}, t\right)=m(t)$. Since the support of $u$ is contained in some ball depending only on $t_{0}>0$ for $t \in\left(0, t_{0}\right)$, the existence of $x_{t}$ is trivial. By a Lipschitz bound in Lemma 3.4, we see that

$$
\frac{u(x, t)}{t} \geq \frac{u(x, t)-u\left(x_{t}, t\right)}{t}+\frac{m(t)}{t} \geq-K \frac{\left|x-x_{t}\right|}{t}+\frac{m(t)}{t} .
$$

If we admit that $x_{t}$ is in the convex hull of $\operatorname{supp} r$ as stated in the next lemma, we take $T$ large such that $m(t) / t \geq R-\varepsilon / 2$ for $t>T$ and $2 K \rho_{1} /$ $T<\varepsilon / 2$ to get $u(x, t) / t \geq R-\varepsilon$ for $t>T, x \in B_{\rho_{1}}$. Note that almost the same argument is found in the proof of [1, Theorem 10.2].

Lemma 3.7. Assume the same hypotheses of Theorem 1.2. Let $S$ denote the convex hull of $\operatorname{supp} r$. Then $\max _{S} u(\cdot, t) \geq \sup _{S^{c}} u(\cdot, t)$, where $S^{c}$ denotes the complement of $S$ in $\mathbf{R}^{N}$. In particular, $x_{t} \in S$. 
This is nontrivial because $x_{t}$ may not be a maximum point of $r$ which is quite different from the first-order case. Such a difference essentially comes from the monotonicity of the geometric flow in the first-order case which determines the way of spreading. To see that $x_{t}$ may not be a maximum point of $r$ for the second-order case, it suffices to consider $r=$ $\left(1_{B_{\rho_{1}}(0)}+\frac{1}{2} 1_{B_{\rho_{2}}(q)}\right) * \eta_{\varepsilon}$ in $\mathbf{R}^{2}$ for isotropic case $a=\sigma=1, \gamma(p)=|p|=$ $M(p)$ with $\rho_{1}<\rho_{*}-\varepsilon, 1=\rho_{*}<\rho_{2}<2-\varepsilon, \varepsilon \in(0,1)$ and $|q|=3$. From the observation for radial case the effect of $1_{B_{\rho_{1}}(0)}$ will eventually negligible for large time and the maximum is taken in $B_{\rho_{2}}(q)$. Moreover, $\sup _{S^{c}} u \leq \inf _{S} u$ may not hold.

Proof of Lemma 3.7. We set that $c(t)=\max _{\partial S} u(\cdot, t)$ and

$$
w(x, t)=\left(c(t)-c^{\prime}(t) \rho(x) / \sigma\right)_{+}, x \in S^{c}, t \geq 0
$$

with

$$
\rho(x)=d_{W_{M}}(x, S):=\inf \left\{W_{M}(x-y) \mid y \in S\right\} .
$$

Formally, it is clear that $w$ solves $u_{t}-\sigma M(-\nabla u)=0$ in $S^{c} \times(0, \infty)$ with $w=c(t)$ on $\partial S$ provided that the time derivative $c^{\prime} \geq 0$. This can be proved rigorously as in Proposition 2.1 and Proposition 2.2. Since $u_{t} \geq 0$, we see that $c^{\prime} \geq 0$. Since $S$ is convex, so is $\rho$. Thus $w$ is a viscosity supersolution of (1.7) in $S^{c} \times(0, \infty)$. By a comparison principle (see e.g. [18]), we see that $u \leq w$ in $S^{c} \times(0, \infty)$. This yields the desired result.

\section{AsYmptotic PROFILE}

4.1. Limit equations. We shall prove Theorem 1.4 in the second-order case. A stronger result for the first-order model is stated as Theorem 2.3.

Proof of Theorem 1.4. As in the first-order case, we may assume that the origin is contained in the interior of $\operatorname{supp} r$. As in the first-order case, $u^{\lambda}(x, t)=u(\lambda x, \lambda t) / \lambda$ has uniform Lipschitz bound (Lemmas 3.3, 3.4) in space-time, for each subsequence of $\lambda \rightarrow \infty$ there is a converges subsequence $u^{\lambda}$ and a limit $v$ such that $u^{\lambda_{j}} \rightarrow v$ uniformly. Moreover, by the stability of viscosity solution $v$ must solve (2.3) outside the origin. Note that the second-order term disappears. At the origin by Theorem 1.2

$$
u^{\lambda}(0, \lambda t) / \lambda \rightarrow R t \quad \text { as } \quad \lambda \rightarrow \infty
$$

locally uniformly in $t$. Thus $v(0, t)=R t$. Since it is not difficult to show that

$$
w(x, t)=R\left(t-W_{M}(x)\right)_{+}
$$

is the unique Lipschitz solution of (2.3) outside the origin with the Dirichlet boundary condition $w(0, t)=R t$, we conclude that $v=w$ and the convergence becomes full convergence. The proof is now complete.

4.2. Case of intermediate speed. Note that our limit function satisfies (2.3) but it is not an envelope solution if $R<c$. Our Theorem 1.3 actually shows that there is an intermediate case. 
Proof of Theorem 1.3. We know by [22] that there is an intermediate case for the maximal solution of

$$
u_{t}-|\nabla u|(\operatorname{div}(\nabla u /|\nabla u|)+1)=c 1_{E_{\ell}}
$$

if $E_{\ell} \subset \mathbf{R}^{2}$ is a square of edge length $2 \ell$ with $\ell \in(1 / \sqrt{2}, 1)$. For given $d$ in Theorem 2.3, we take $\varepsilon>0$ small so that $1_{E_{\ell_{1}}} \geq r_{\varepsilon} \geq 1_{E_{\ell_{2}}}$ so that $\ell_{2}<d<$ $\ell_{1}$ and $\ell_{i} \in(1 / \sqrt{2}, 1) \quad(i=1,2)$. Let $u_{i}$ be the maximal solution of (4.1) with initial data $\left.u_{i}\right|_{t=0}=0$ and $\ell=\ell_{i}(i=1,2)$. We know $\lim \sup _{t \rightarrow \infty} u_{1} /$ $t \leq c_{1}<c, \liminf _{t \rightarrow \infty} u_{2} / t \geq c_{2}>0$ by [22]. By comparison, $u_{1} \leq u \leq u_{2}$ thus $c_{2} \leq R_{\varepsilon} \leq c_{1}$ for sufficiently small $\varepsilon$.

4.3. Asymptotic profile of large level set. We shall give a simple proof for Theorem 1.6 based on Theorem 1.4. Let $\left\{E_{t}\right\}_{t \geq 0}$ be an increasing family of bounded closed sets which exhausts $\mathbf{R}^{N}$, i.e., for any compact set $K$ there is $t$ such that $K \subset E_{t}$. If $\left\{E_{t}\right\}$ is exhaustive,

$$
q\left(x, E_{0}\right)=\inf \left\{t \geq 0 \mid x \in E_{t}\right\} .
$$

is well defined for all $x \in \mathbf{R}^{N}$. It is continuous if $E_{t}$ is continuous in $t$ in Hausdorff distance sense and strictly monotone in the sense that $E_{t} \subset \inf E_{s}$ for $s>t \geq 0$.

For a given bounded closed set $E_{0}$, let $S_{t}$ be a level-set flow of (1.4) starting from $S_{0}=\partial E_{0}$. As is in [18], we say that the open set $D_{t}$ enclosed by $S_{t}$ is called an open evolution while $E_{t}=D_{t} \cup S_{t}$ is called a closed evolution starting, respectively, $D_{0}$ and $E_{0}$.

Lemma 4.1. Let $E_{0}=\kappa \mathcal{W}_{\gamma}$ with $\kappa>\rho_{*}(=a(N-1) / \sigma)$. Let $E_{t}$ be the closed evolution of (1.4) starting from $E_{0}$. Then the function $w_{\kappa}(x, t)=(t-q(x))_{+}$ with $q=q\left(x, E_{0}\right)$ is a viscosity solution of (1.8) with $r=1_{E_{0}}$ and $\left.w\right|_{t=0}=0$.

Proof. It is easy to see that $w$ is a viscosity solution of (1.8) once $q$ is a well-defined continuous function. Since initially $E_{0} \subset \inf E_{s}$ for $s>0$, the strict monotonicity $E_{t_{1}} \subset \operatorname{int} E_{t_{2}}$ for $t_{1}<t_{2}$ is clear by comparison. Upper semicontinuity of $E_{t}$ is trivial and left lower semicontinuity follows from a general theory [18, Theorem 4.5.5]. The right lower semicontinuity follows from the monotonicity, so $E_{t}$ is continuous in $t$ in the Hausdorff distance sense.

To show that $E_{t}$ is exhaustive, we compare with a special solution of $V=$ $m_{0} \gamma\left(a H_{\gamma}+\sigma\right)$ such that a constant $m_{0}>0$ is taken so that $M(p) \geq m_{0} \gamma(p)$ for $p \in \mathbf{R}^{N}$. Since this equation has a self-similar growing solution [37], [26] of the form $\lambda(t) W_{\gamma}$ with $\lambda(t) \rightarrow \infty$ as $t \rightarrow 0$ and since such a solution is a subsolution of of (1.4) in the level-set sense, by comparison $\left\{E_{t}\right\}$ is exhaustive.

Proof of Theorem 1.6. We shall prove that

$$
\lim _{t \rightarrow \infty} \frac{q(t x)}{t}=W_{M}(x)
$$

locally uniformly in $x \in \mathbf{R}^{N}$. There is a Lipschitz function $r$ such that $0 \leq$ $r \leq 1$ and the set $\{r=1\}$ equals $\kappa^{\prime} W_{\gamma}, \kappa^{\prime}>\rho_{*}, \kappa^{\prime}<\kappa$ and $\operatorname{supp} r \subset \kappa W_{\gamma}$. By comparison, it is clear that

$$
w_{\kappa^{\prime}}(x, t) \leq u(x, t) \leq w_{\kappa}(x, t),
$$


where $u$ is the solution of (3.1) with zero initial data. The estimate $w_{\kappa^{\prime}} \leq u$ implies that the asymptotic speed of $u$ must be one. By Theorem 1.4 and $u \leq w_{\kappa}$, we see that

$$
\lim _{t \rightarrow \infty} \frac{u(t x, t)}{t}=\left(1-W_{M}(x) / \sigma\right)_{+} \leq\left(1-\lim \sup _{t \rightarrow \infty}^{*} q(t x) / t\right)_{+},
$$

where lim sup* is a relaxed limit, i.e., it is defined as

$$
\lim \sup _{t \rightarrow \infty}^{*} f(t, x)=\lim _{t \rightarrow \infty} \sup \{f(s, y)|s \geq t,| y-x \mid \leq 1 / t\} .
$$

This in particular implies that

$$
\lim \sup _{t \rightarrow \infty}^{*} \frac{q(t x)}{t} \leq W_{M}(x) / \sigma \quad \text { for } \quad x \quad \text { satisfying } \quad W_{M}(x) \leq 1 .
$$

The other estimate is easy. It is easy to see that $(\sigma t+\beta) \partial \mathcal{W}_{M}$ with $\beta>0$ is a level-set supersolution of (1.4) (which is a solution of (1.2)). We take $\beta$ large enough so that $\beta \mathcal{W}_{M}$ includes $\kappa \mathcal{W}_{\gamma}$. By comparison, $E_{t} \subset(\sigma t+\beta) \mathcal{W}_{M}$ since $E_{0}=\kappa \mathcal{W}_{\gamma}$. This implies

$$
\liminf _{* t \rightarrow \infty} \frac{q(t x)}{t} \geq W_{M}(x) / \sigma,
$$

where $\lim \inf _{* t \rightarrow \infty} f=-\lim \sup _{t \rightarrow \infty}^{*}(-f)$. This implies $\lim _{t \rightarrow \infty} \frac{q(t x)}{t}=W_{M}(x) /$ $\sigma$ locally uniformly since $W_{M}(x)$ is positively homogeneous of degree one.

The estimate (4.2) implies that for any $\varepsilon>0$

$$
\sigma \mathcal{W}_{M, \varepsilon} \subset t^{-1} D_{t}
$$

for sufficiently large $t$, where $\mathcal{W}_{M, \varepsilon}=\left\{x \in \mathcal{W}_{M} \mid \operatorname{dist}\left(x, \mathcal{W}_{M}^{c}\right)>\varepsilon\right\}$. Note that there is no fattening in this setting (1.8) so that int $E_{t}=D_{t}$. The estimate $E_{t} \subset(\sigma t+\beta) \mathcal{W}_{M}$ implies

$$
t^{-1} E_{t} \subset \sigma \mathcal{W}_{M}^{\varepsilon}
$$

for sufficiently large $t$, where $\mathcal{W}_{M}^{\varepsilon}=\left\{x \in \mathbf{R}^{n} \mid \operatorname{dist}\left(x, \mathcal{W}_{M}\right)<\varepsilon\right\}$.

For general $\Gamma_{0}$, we compare with $\kappa \mathcal{W}_{\gamma}$ and $\kappa^{\prime} \mathcal{W}_{\gamma}$ so that $\kappa \mathcal{W}_{\gamma} \supset \Gamma_{0}$ or $\Gamma_{0}$ encloses $\kappa^{\prime} \mathcal{W}_{\gamma}$ for a suitable choice of $\kappa, \kappa^{\prime}>\rho_{*}$. The desired results like (4.3) and (4.4) for this initial data follows from comparison principle and behavior of solutions starting from $\kappa \mathcal{W}_{\gamma}$ or $\kappa^{\prime} \mathcal{W}_{\gamma}$.

Remark 4.2. In [29, Theorem 6.1], a more general equation like

$$
V=v_{1}(\mathbf{n}, \mathbf{A})+\sigma M(\mathbf{n}) \quad \sigma>0, M>0
$$

is handled under the assumptions that $v_{1}$ is monotone nondecreasing in the second fundamental form $\mathbf{A}$ in the direction of $\mathbf{n}$ and positively homogeneous of degree one in $\mathbf{A}$, i.e., $v_{1}(\mathbf{n} \lambda \mathbf{A})=\lambda v_{1}(\mathbf{n}, \mathbf{A}), \lambda>0$ not necessarily linear. This case can be handled in our setting. The crucial step is to obtain a Lipschitz bound where we have used

$$
-\sum_{i, j} \frac{\partial F}{\partial X_{i j}} X_{i j}=u_{t}-\sigma M(-\nabla u)-r .
$$

Fortunately, this equality still holds if $v_{1}$ satisfies the Euler equation, i.e., $\sum_{i} \frac{\partial f}{\partial p_{i}} p_{i}=f$ for homogeneous functions. 


\section{UnSCALED ASYMPTOTIC PROFILE}

5.1. Large time convergence of a solution. We next try to find an unscaled asymptotic profile in the sense that we seek a function $w$ such that for any ball $B$

$$
\sup _{x \in B}|u(x, t)-R t-w(x)| \rightarrow 0,
$$

as $t \rightarrow \infty$. Here, $(w, R)$ satisfies a stationary problem $R+F\left(\nabla w, \nabla^{2} w\right)=$ $r(x)$ in $\mathbf{R}^{n}$. We emphasize here that, in general, solutions to this stationary problem are not unique even up to additive constants. See examples in [30, Chapter 6] for instance. Therefore, the convergence (5.1) is not trivial in general. Such a problem is well studied in the first order model. It was started by [31] and [12]. The problem is especially well studied for the Hamilton-Jacobi equations $u_{t}+H(x, \nabla u)=0$ for convex Hamiltonian $H$ in a periodic setting. For $\mathbf{R}^{N}$ setting, see the work of H. Ishii [28]. These results are based on approach by dynamical systems. There is a PDE approach by G. Barles and P. E. Souganidis [2] which covers some class of non convex Hamiltonian. However, for the second-order problems less is known especially parabolicity is degenerated. Recently, nonlinear adjoint method introduced by L. C. Evans [10] is adjusted to apply such a kind of problems of large time behavior by F. Cagnetti, D. Gomes, H. Mitake and H. V. Tran [4]. This method allows some degenerate second order term but it does not apply to our second model because the degeneracy depends on a solution. The reader is referred to a recent nice survey [30] for more details and references.

For the solution $u$ to the initial value problem of (3.1), what we know is that $u(x, t)-R t$ converges locally uniformly as $t \rightarrow \infty$ to some function $w$ by taking a subsequence because of Lipschitz bound. Moreover, $w$ solves $R+F\left(\nabla w, \nabla^{2} w\right)=r(x)$. However, such an equation is not well studied even under periodic setting. Therefore, the full convergence (5.1) is not yet known.

We finally point out that the asymptotic speed $R$ is independent of the choice of initial data but the profile may depend on the initial data in a nonlinear way. Therefore, a key question here could be how $w$ depends on $u_{0}$. For the first order case with a convex Hamiltonian, a representation formula for $w$ is given by A. Davini and A. Siconolfi [9], where the values of initial data on the Aubry set and the infimum stability of viscosity solutions essentially play a role. In second order case, this question is rather open even in case when the equation is linear in $\nabla^{2} w$. Also, in first order case, if the Hamiltonian is non-convex, then it is hard to study the structure of the above stationary problem and it is rather open, as the weak KAM theory does not work well under such situation.

5.2. Some open problems. We conclude this paper to give a couple of open problems.

Problem 1. Show the full convergence (5.1) even if the equation is isotropic like

$$
u_{t}-(\operatorname{div}(\nabla u /|\nabla u|)+1)|\nabla u|=r(x) .
$$


Study the uniqueness set for equation $R+F\left(\nabla w, \nabla^{2} w\right)=r(x)$, the dependence of $w$ in (5.1) on the initial data $u_{0}$ in the case of second order equations.

Problem 2. Show the existence of $R$ when $r$ is discontinuous. Study how $R$ depends on $r$ both qualitatively and quantitively.

In crystal growth problems, it is important to know how the growth rate depends on configuration of sources, i.e., geometric configuration of $E$ when $r=c(x) 1_{E}$ with some positive function depending on $x$; see [33], [34] for spiral growth. Several examples are studied in [23].

Problem 3. How regular is the solution $u$ when $r \geq 0$ is regular and initial data is zero?

These problems are very natural goals to derive unscaled asymptotic profile. The next problems are related to crystalline flow.

Problem 4 (Crystalline flow). Prove that if $u_{j}$ is a viscosity solution of (3.1) with crystalline $\gamma$, so is its locally uniform limit $u$ as $j \rightarrow \infty$.

This is only proved in $N=2$ in [14]. The problem for $N \geq 3$ is that definition in [24], [25] is not stable under such a limiting procedure. Once this is settled, an explicit solution given in Theorem 3.2 is also the maximal viscosity solution for crystalline case when $N \geq 3$.

Problem 5. It seems that the spatially Lipschitz bound should be true for crystalline spreading law. Extend Lemma 3.4 to crystalline case.

If so, this would yield the existence of the asymptotic speed for crystalline case.

We conclude this paper by pointing out that there are several potential applications of birth and spread models to other fields not limited in the field of crystal growth by considering various spreading laws. In this paper, we consider the spreading law $V=M(\mathbf{n})\left(a H_{\gamma}+\sigma\right)$ but it is interesting to consider more general spreading law as $V=g\left(\mathbf{n}, H_{\gamma}\right)$. For example, in [23] formation of volcano profile is explained by taking inverse curvature like flow as the spreading law. It is worth to study above problems in these more general setting.

\section{ACKNOWLEDGMENTS}

The author is grateful to Professor Hiroyoshi Mitake for valuable comments. This work was partly supported by Japan Society for the Promotion of Science (JSPS) through grants No. 26220702 (Kiban S), No. $17 \mathrm{H} 01091$ (Kiban A) and No. 16H03948 (Kiban B).

\section{REFERENCES}

[1] G. Barles, An introduction to the theory of viscosity solutions for first-order HamiltonJacobi equations and applications. Hamilton-Jacobi equations: approximations, numerical analysis and applications, 49-109, Lecture Notes in Math., 2074, Fond. CIME/CIME Found. Subser., Springer, Heidelberg, 2013.

[2] G. Barles and P. E. Souganidis, On the large time behavior of solutions of HamiltonJacobi equations. SIAM J. Math. Anal. 31 (2000), 925-939. 
[3] W. K. Burton, N. Cabrera and F. C. Frank, The growth of crystals and the equilibrium structure of their surfaces. Philos. Trans. Roy. Soc. London. Ser. A. 243 (1951), 299-358.

[4] F. Cagnetti, D. Gomes, H. Mitake and H. V. Tran, A new method for large time behavior of degenerate viscous Hamilton-Jacobi equations with convex Hamiltonians. Ann. Inst. H. Poincaré Anal. Non Linéaire 32 (2015), 183-200.

[5] A. Chambolle, M. Morini, M. Novaga and M. Ponsiglione, Existence and uniqueness for anisotropic and crystalline mean curvature flows. available at https://arxiv.org/abs/1702.03094

[6] A. Chambolle, M. Morini and M. Ponsiglione, Existence and uniqueness for a crystalline mean curvature flow. Comm. Pure Appl. Math. (2016), DOI: 10.1002/cpa.21668

[7] Y.-G. Chen, Y. Giga and S. Goto, Uniqueness and existence of viscosity solutions of generalized mean curvature flow equations. J. Differential Geom. 33 (1991), 749-786.

[8] M. G. Crandall, H. Ishii abd P.-L. Lions, User's guide to viscosity solutions of second order partial differential equations. Bull. Amer. Math. Soc. (N.S.) 27 (1992), 1-67.

[9] A. Davini and A. Siconolfi, A generalized dynamical approach to the large time behavior of solutions of Hamilton-Jacobi equations. SIAM J. Math. Anal. 38 (2006), $478-502$.

[10] L. C. Evans, Adjoint and compensated compactness methods for Hamilton-Jacobi PDE. Arch. Ration. Mech. Anal. 197 (2010), 1053-1088.

[11] L. C. Evans and J. Spruck, Motion of level sets by mean curvature. I. J. Differential Geom. 33 (1991), 635-681.

[12] A. Fathi, Sur la convergence du semi-groupe de Lax-Oleinik. C. R. Acad. Sci. Paris Sér. I Math. 327 (1998), 267-270.

[13] M.-H. Giga and Y. Giga, Evolving graphs by singular weighted curvature. Arch. Rational Mech. Anal. 141 (1998), 117-198.

[14] M.-H. Giga and Y. Giga, Generalized motion by nonlocal curvature in the plane. Arch. Ration. Mech. Anal. 159 (2001), 295-333.

[15] M.-H. Giga and Y. Giga, On the role of kinetic and interfacial anisotropy in the crystal growth theory, Interfaces and Free Boundaries 15 (2013), 429-450.

[16] M.-H. Giga, Y. Giga and N. Požár, Anisotropic total variation flow of non-divergence type on a higher dimensional torus. Adv. Math. Sci. Appl. 23 (2013), 235-266.

[17] M.-H. Giga, Y. Giga and N. Požár, Periodic total variation flow of non-divergence type in $\mathbb{R}^{n}$. J. Math. Pures Appl. (9) 102 (2014), 203-233.

[18] Y. Giga, Surface evolution equations. A level set approach. Monographs in Mathematics, 99. Birkhäuser Verlag, Basel, 2006. (earlier version: Lipschitz Lecture Notes 44, University of Bonn, 2002.)

[19] Y. Giga and N. Hamamuki, Hamilton-Jacobi equations with discontinuous source terms. Comm. Partial Differential Equations 38 (2013), 199-243.

[20] Y. Giga, Q. Liu and H. Mitake, Large-time asymptotics for one-dimensional Dirichlet problems for Hamilton-Jacobi equations with noncoercive Hamiltonians. J. Differential Equations 252 (2012), 1263-1282.

[21] Y. Giga, Q. Liu and H. Mitake, Singular Neumann problems and large-time behavior of solutions of noncoercive Hamilton-Jacobi equations. Trans. Amer. Math. Soc. 366 (2014), 1905-1941.

[22] Y. Giga, H. Mitake and H. V. Tran, On asymptotic speed of solutions to level-set mean curvature flow equations with driving and source terms. SIAM J. Math. Anal. 48 (2016), 3515-3546.

[23] Y. Giga, H. Mitake, T. Ohtsuka and H. V. Tran, in preparation.

[24] Y. Giga and N. Požár, A level set crystalline mean curvature flow of surfaces. Adv. Differential Equations 21 (2016), 631-698.

[25] Y. Giga and N. Požár, Approximation of general facets by regular facets with respect to anisotropic total variation energies and its application to the crystalline mean curvature flow. Comm. Pure Appl. Math., to appear. 
[26] M. E. Gurtin, Thermomechanics of evolving phase boundaries in the plane. Oxford Mathematical Monographs. The Clarendon Press, Oxford University Press, New York, 1993.

[27] N. Hamamuki, On large time behavior of Hamilton-Jacobi equations with discontinuous source terms. Nonlinear analysis in interdisciplinary sciences-modellings, theory and simulations, 83-112, GAKUTO Internat. Ser. Math. Sci. Appl., 36, Gakkōtosho, Tokyo, 2013.

[28] H. Ishii, Asymptotic solutions for large time of Hamilton-Jacobi equations in Euclidean n space. Ann. Inst. H. Poincaré Anal. Non Linéaire 25 (2008), 231-266.

[29] H. Ishii, G. E. Pires and P. E. Souganidis, Threshold dynamics type approximation schemes for propagating fronts. J. Math. Soc. Japan 51 (1999), 267-308.

[30] H. Mitake and H. V. Tran, Dynamical properties of Hamilton-Jacobi equations via the nonlinear adjoint method: Large time behavior and Discounted approximation. Dynamical and Geometric Aspects of Hamilton-Jacobi and Linearized Monge-Ampère Equations, VIASM 2016 (H. Mitake and H. V. Tran eds.), Lecture Note in Math. 2183, Springer 2017, 125-228.

[31] G. Namah and J.-M. Roquejoffre, Remarks on the long time behaviour of the solutions of Hamilton-Jacobi equations. Comm. Partial Differential Equations 24 (1999), 883893.

[32] M. Ohara and R. C. Reid. Modeling crystal growth rates from solution, PrenticeHall, Englewood Cliffs. NJ 1973.

[33] T. Ohtsuka, Y.-H. R. Tsai and Y. Giga, A level set approach reflecting sheet structure with single auxiliary function for evolving spirals on crystal surfaces. J. Sci. Comput. 62 (2015), 831-874.

[34] T. Ohtsuka, Y.-H. R. Tsai and Y. Giga, Growth rate of crystal surfaces with several dislocation centers. earlier version: Hokkaido University Preprint Series in Mathematics \#1099 (2017).

[35] T. P. Schulze and R. V. Kohn, A geometric model for coarsening during spiral-mode growth of thin films. Phys. D 132 (1999), 520-542.

[36] P. Smereka, Spiral crystal growth. Phys. D 138 (2000), 282-301.

[37] H. M. Soner, Motion of a set by the curvature of its boundary. J. Differential Equations 101 (1993), 313-372.

[38] J. Xin and Y. Yu, Sharp asymptotic growth laws of turbulent flame speeds in cellular flows by inviscid Hamilton-Jacobi models. Ann. Inst. H. Poincaré Anal. Non Linéaire 30 (2013), 1049-1068.

[39] J. Xin and Y. Yu, Asymptotic growth rates and strong bending of turbulent flame speeds of G-equation in steady two-dimensional incompressible periodic flows. SIAM J. Math. Anal. 46 (2014), 2444-2467.

[40] E. Yokoyama Y. Giga and P. Rybka, A microscopic time scale approximation to the behavior of the local slope on the faceted surface under a nonuniformity in supersaturation. Phys. D 237 (2008), 2845-2855.

Graduate School of Mathematical Sciences, University of Tokyo, 3-8-1

Komaba, Meguro-KU, TOKYO 153-8914, JAPAN

E-mail address: labgiga@ms.u-tokyo.ac.jp 